\title{
Power Quality Prolems and Improvement Techniques
}

\author{
${ }^{1}$ Abdul-Jabbar Fathel Ali, ${ }^{2}$ Wael Hussein Zayer and ${ }^{1}$ Samhar Saeed. Shukir \\ ${ }^{1}$ Electrical Department, College of Engineering, University of Wasit, Kut, Iraq \\ ${ }^{2}$ Electronic Department, Amara Technical Institute, Southern Technical University, Besra, Iraq \\ AJF_572@yahoo.com,Wael_zayer@yahoo.com, samharalwandi@gmail.com
}

\begin{abstract}
Recently a large interest has been focused on power quality domain due to: disturbances caused by the non-linear loads, increase in the number of electronic devices and growth of renewable energy sources. Power quality measures the efficiency of electric power transmitted from generation to the industrial, domestic and commercial consumers. At least $50 \%$ of power quality problems are of voltage quality type. In a power system voltage sags and voltage distortion introduced by harmonics are considered to be the most severe affecting power quality because of both utilities and consumers are affected by these disturbances. These problems could be solved by: design equipments and electrical systems to prevent electrical disturbances from causing equipments or systems to malfunction, analyze the symptoms of a power quality problems to determine its causes and solutions, study the medium that is transmitting the electrical disturbance and eliminate or reduce the effect of that medium, treat the symptoms of the power quality problems by using the power conditioning device and custom power device. This study, presents the power quality problems such as sags, swells, harmonics, voltage interruptions and transient and presents power quality enhancement techniques which are two categories power conditioning devices and custom power devices the second method is the most efficient and effective method to solve the power quality problems and protective the sensitive loads from power disturbances. The modeling and simulation of a power distribution system was achieved using MATLAB/Simulink. Three phase fault and double line to ground fault are created with the proposed system and the faults are initiated at a duration of $0.8 \mathrm{sec}$ and keep till $0.95 \mathrm{sec}$. Dynamic Voltage Restorer (DVR) is used to treat the power quality problems.
\end{abstract}

Key words: Non-linear loads, systems malfunction, power quality, study the medium, electrical disturbance, transmitting

\section{INTRODUCTION}

Electrical power is the main element that required in any function in the commercial and industrial sectors, therefore, it should be available at all times. Power quality is a set of parameters that define the characteristics of the power supply as delivered to the consumers in normal operating conditions in terms of continuity of supply and characteristics of voltage such as frequency, magnitude, waveform and symmetry. Recently, power quality is not only a technical problem but also a problem that leads to financial issues. Many surveys have been shown that poor power quality causes large economic losses to industrial sectors. Moreover, large amount of power is wasted due to poor power quality in the services. A survey conducted to assess economic impact of poor power quality faced by various Indian industries by
Emerson group and Manufacturer's Association for Information Technology (MAIT) states the estimated loss in India is close to 10 billion USD in direct losses due to poor power quality and operating environment related to downtime. Awareness among the industries regarding the power quality should be created which helpsin the increase in productivity and Gross Domestic Product (GDP) growth. PQ events are unpredictable but they can be anticipated. Determining the exact problems requires sophisticated electronic test equipment but the symptoms such as equipment failure during lightning and thunder storm, tripping of circuit breakers without being overloaded, automated system stopping for no apparent reason, electronic system failure on a frequent basis and also working of electronic system in one location but not in another location, help us to identify the PQ problems (Babu and Kamaraj, 2017). The power quality is gaining

Corresponding Author: Abdul-Jabbar Fathel Ali, Electrical Department, College of Engineering, University of Wasit, Kut, Iraq, AJF_572@yahoo.com 

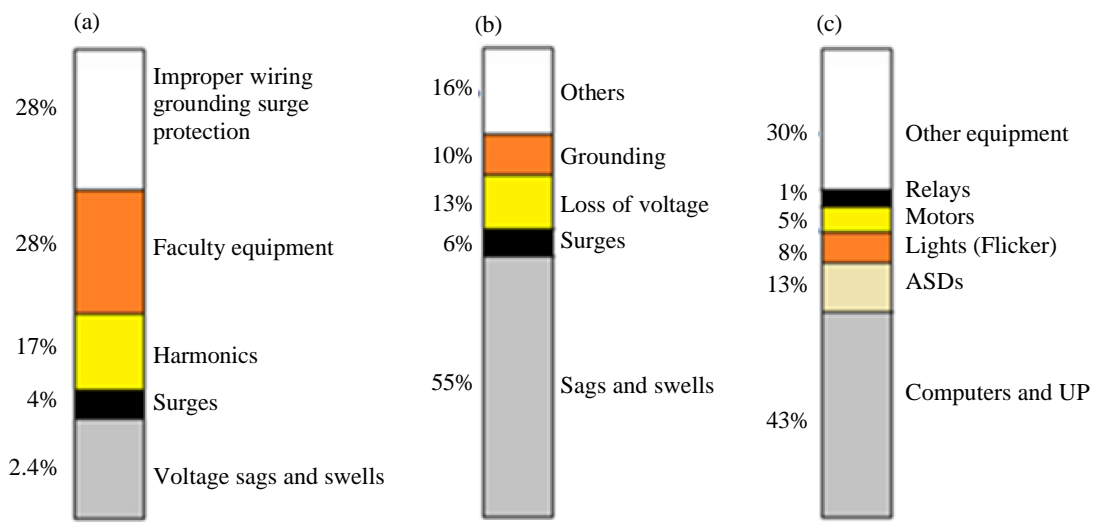

Fig. 1: Basic disturbances; a) Causes at customer side; b) Causes at utility side and c) Affected equipment

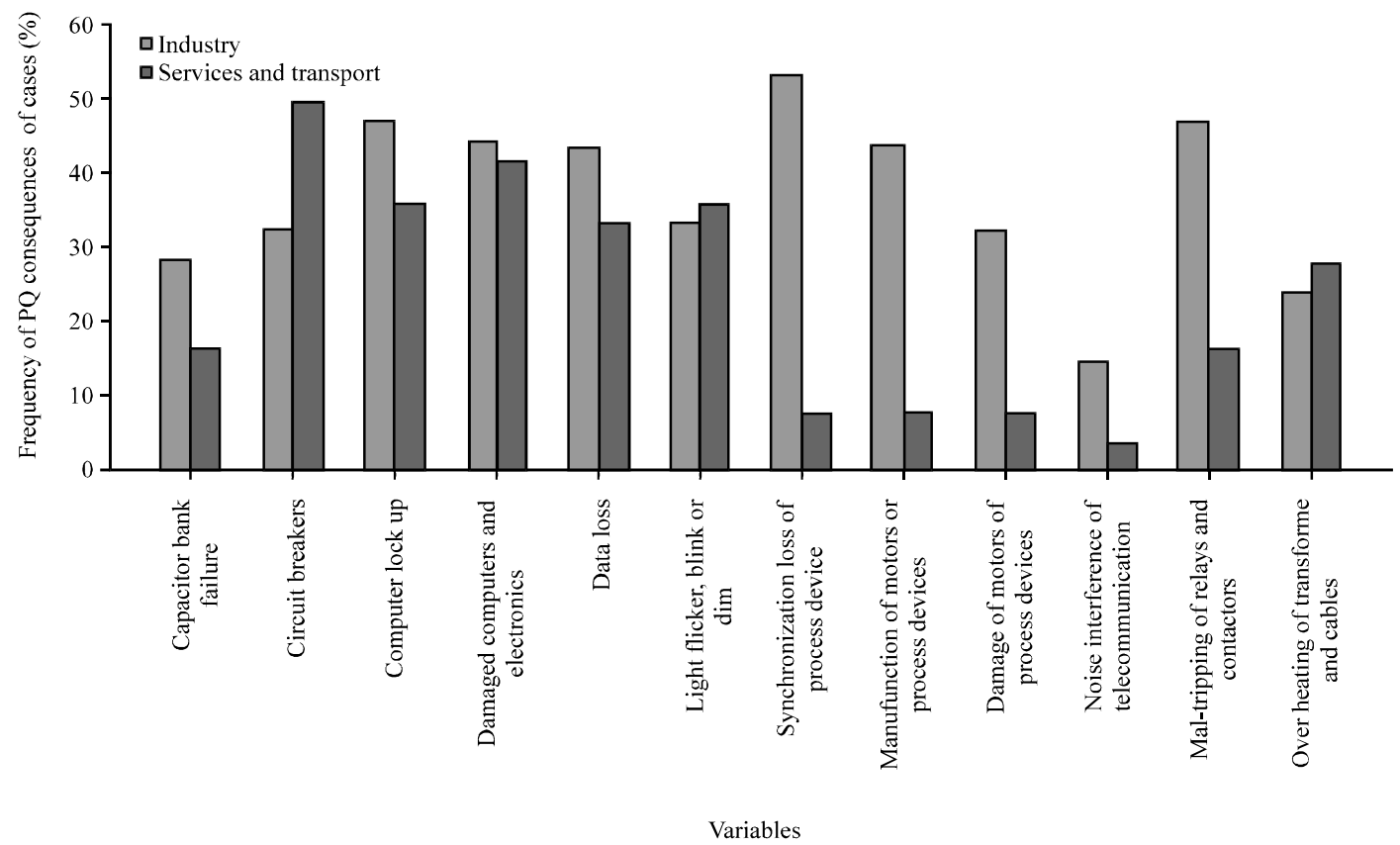

Fig. 2: The consequences of poor power quality as experienced by the consumers

importance from the following reasons: power electronic devices and developed equipment with microprocessor based controls are more sensitive to power quality variations, the use ofpower electronic devices based control of variable speed drives and switched mode power supplies creates a lot of electical disturbances into the supply system (Babu and Kamaraj, 2017). The statistical aspects of the power quality problems as identified a number of surveys are shown in Fig. 1. From this figure the most common causes of disturbances at customers side are: faulty equipment and improper grounding to protect against surge while the most common disturbances at utility side are caused by: sags and swell. And the most common affected equipements are: computers and UP (Fig. 2).

A large number of equipments that consists of power electronics devices are used by the industrial, commercial and residential customers at their installations these equipments are sensitive to power quality disturbances. the studies and the surveys in different countries around the world have been noticed that industries are vulnerable to reliability issue (long and short interruptions). The voltage sag is the main power quality problem for the manufacturing industries and telecom sectors. Harmonics problems are perceived especially by the service sectors 
such as hospitals and banks. By Manson and Targosz (2008), Bhattacharyya and Cobben (2011) another survey has been shown that the main problem. For the continuation of industries are: loss of synchronization of processing devices, the tripping of relays and contactors without overloading and damaged computers and electronics devices. Also, it has been noticed that the main problems of the power quality disturbances in service and transport sectors are: circuit breaker, damaged electronics equipments and lights flicker, blink or dim. All these surveys are shown in the Fig. 2.

\section{MATERIALS AND METHODS}

Classification of power quality problem: Power quality is a combination of voltage and current quality. In most cases the utility is responsible for voltage quality at the Point of Common Coupling (PCC) while the consumers often influencethe current quality at the PCC (Bhattacharyya et al., 2007; Bollen, 2001). There is always a relationship between the voltage and the current in any practical power system (Pohjanheimo, 2003). According to the IEEE definitions from IEEE standard 1159-1995 (Singh and Simina, 2004) Fig. 3 shows the demarcation of various power quality problems.

Another survey is shown in Fig. 4 we can conclude from this figure that the voltage sag, harmonics and asymmetrical voltage are the most common power quality problems in the industrial process.

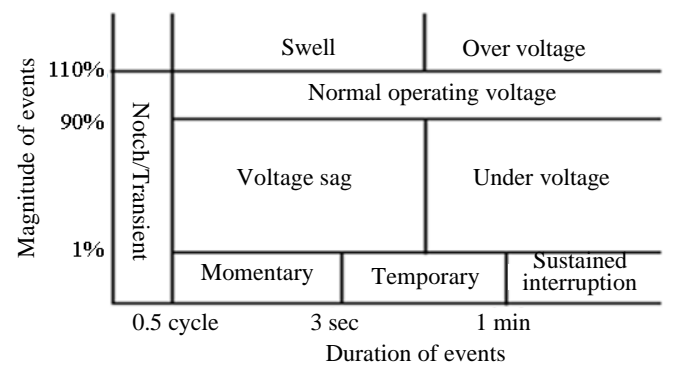

Fig. 3: The demarcation of power quality problems

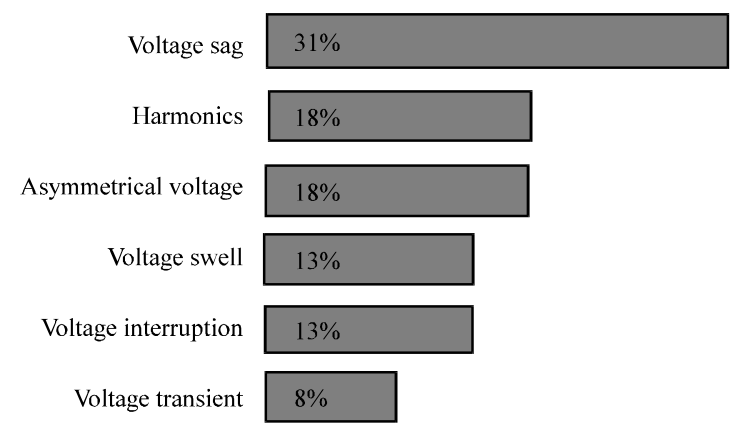

Fig. 4: Classification of power quality problems

\section{Power quality problems}

Voltage sags: A voltage sag is a sudden decrease in the rms voltage that the voltage value become between 0.1 and 0.9 p.u from its nominal value and lasting from 0.5 cycle to several seconds. Sags with duration of less than a 0.5 cycle are considered as transients. Voltage sag either symmetrical or unsymmetrical as shown in Fig. 5 and 6.

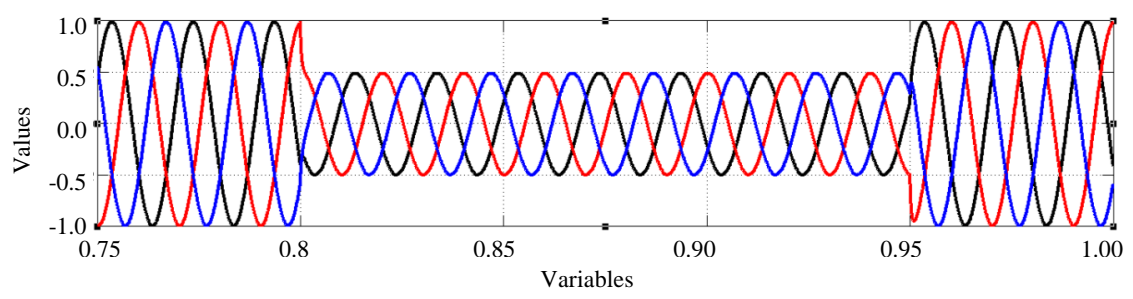

Fig. 5: Symmetrical sag caused by three phase fault

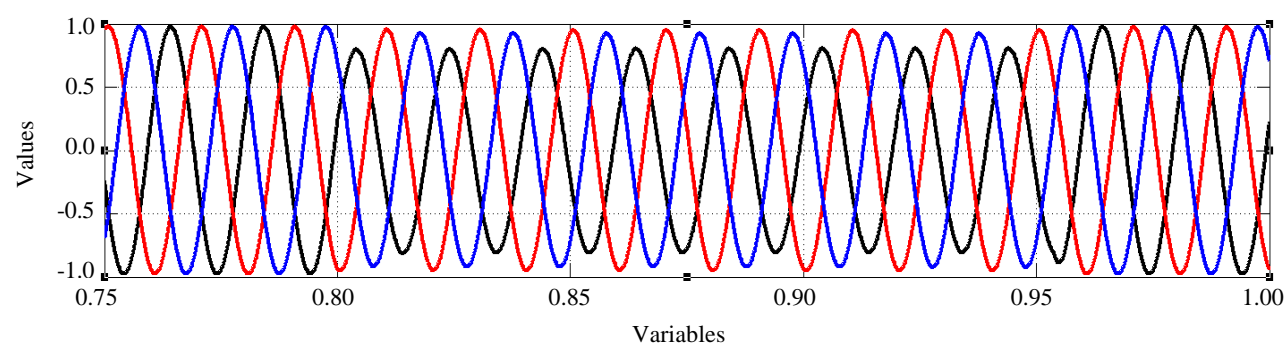

Fig. 6: Usymmetrical sag caused by double line fault 


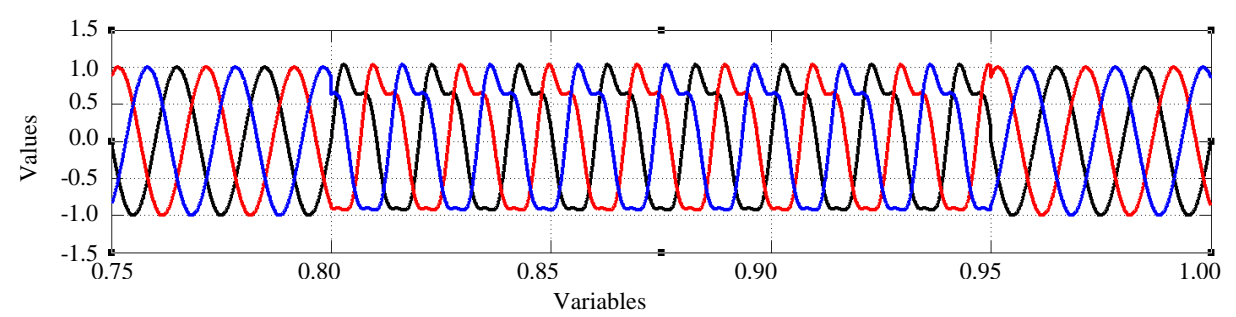

Fig. 7: Voltage harmonics

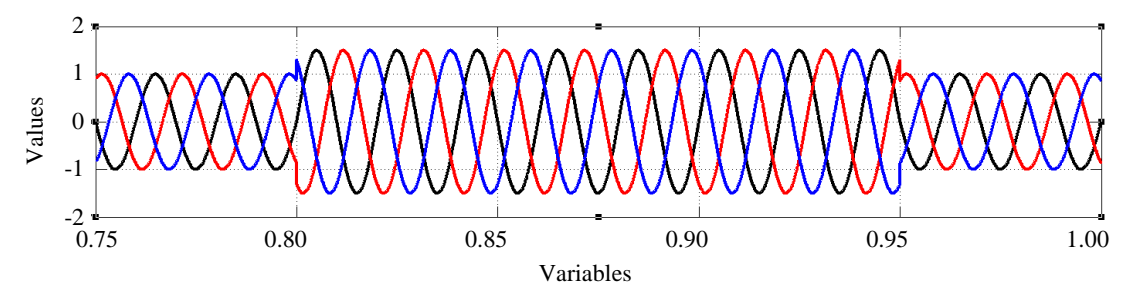

Fig. 8: Voltage swell

Harmonics: Harmonics can be defined as the spectral components at frequencies that are integer multiples of the fundamental frequency. Figure 7 the 2 nd order harmonic and the 3rd order harmonic are presence with the fundamental frequency waveform. It looks distorted as shown in Fig. 7 The main cause to harmonic voltage distortion is the nonlinear loads. Also, there are several factors that contributing to the voltage harmonics such as:

- The voltage generated by a synchronous machine is not exactly sinusoidal due to small deviations from the ideal shape of the machine

- The power system transmitting the electrical energy from the generation stations to the load is not completely linear. For example is the power transformer where the non-linearity is due to saturation of the magnetic flux in the iron core of the transformer

- Modren example of a non-linear power system component is the High Voltage Direct Current (HVDC) link. The transformation from AC-DC and back takes place by using power-electronics components which only conduct during part of a cycle

The most common harmonic current drawn non-linear loads are all single and three phase power converters which contain rectifiers such as DC motor drives, Adjustable Speed Drives (ASD), Uninterruptable Power Supplies (UPS), Switched Mode Power Supplies (SMPS), fluorescent lighting, lectrical heating furnaces, welding machine arc furnaces. Besides these non-linear loads, AC generator, $\mathrm{AC}$ motors and transformers also produce harmonic currents. The effects of harmonics include harmonic heating and torque pulsation. These effects ultimately result in damage to the equipment. Transformers and other industrial equipment are more susceptible to harmonics. Harmonics can lead to control errors and malfunction of equipment. This can especially, be a big problem in industrial power systems where there is a large concentration of distorting load as well as sensitive load (Chan et al., 1999).

Swell: A voltage swell is a sudden increase in the rms voltage between 1.1 and 1.9 p.u at the point in the electrical system and lasting for 0.5 cycle to several seconds. Swells with duration of less than a cycle are regarded as transients. Figure 8 shows a waveform depicting a voltage swell.

Voltage interruption: The voltage is decreased to $<0.1 \mathrm{pu}$ for a period not exceeding $1 \mathrm{~min}$ as demonstrated in Fig. 9.

Transient: Transient is a sudden change in steady state condition of voltage, current or both as depicted in Fig. 10.

Asymmetrical voltage: Asymmetrical voltage occurs in the case of three-phase voltages where the values ofsingle phase voltages are not equal in magnitude or the phase angle difference between these voltages is not equal. Asymmetrical voltage occurs due to a large single loads such as inductive furnaces and also occurs due to the unbalanced loading of each of the three phases. Also, unbalance fault causes asymmetrical voltage as shown in Fig. 6. 


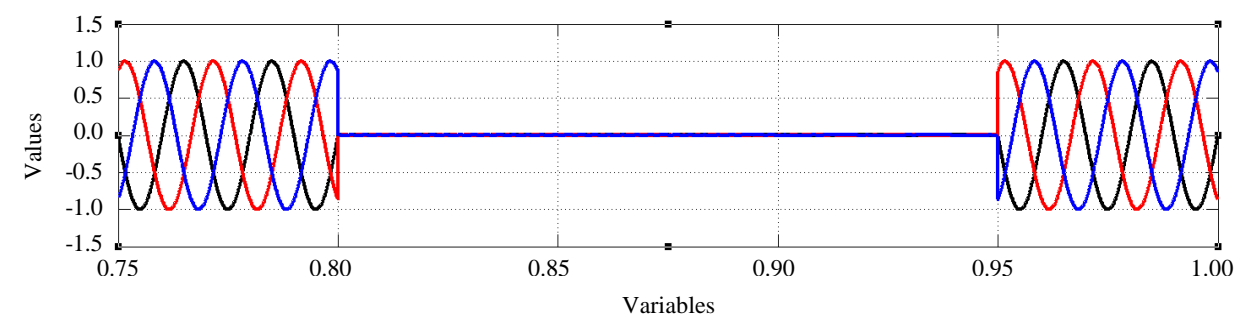

Fig. 9: Voltage interruption

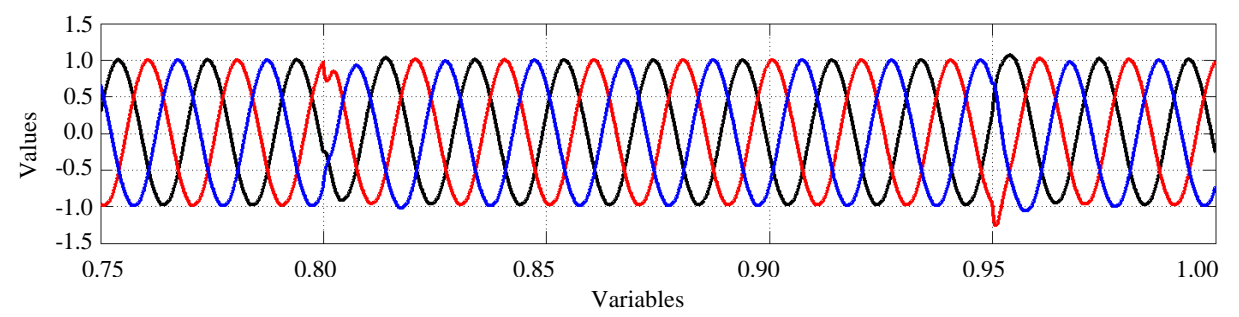

Fig. 10: Voltage transient

Effects of voltage sags: The problem of sag voltage is the most serious problem among the problems of power quality where it leads to the flow of high current and thus, damage sensitive devices such as: computers, Programmable Logic Controller (PLC), controller power supplies, Adjustable Speed Drives (ASD), motor starter contactors. The voltage sag problems in industrial equipment include (Bhanoo, 1998):

- Relays opening without overloading because of the sag affecting the relay's coil voltage

- Sensor voltage gives incorrect reports such as water pressure sensors or air flow sensors

- Circuit breaker or fuses operating due to the large increase in current immediately after the sag

\section{Causes of voltage sag}

Voltage sag due to faults: Voltage sags are mostly because of the power system faults. The severity of sag depends on the type of fault and the distance from the source and the fault, a three-phase fault near a distribution substation leads to all the customers connected to these substation will face deep sag (Ghosh and Ledwich, 2002). The main reasons for power system fault are: weather (snow, wind, lightning) and interference from smaller animals and birds.

Reclosure of circuit breaker: Operation of a circuit breaker or reclosure causes a temporary fault of a specific line. In case of weak grid, voltage sag in the neighboring lines will be occurred because of this temporary fault. The extent of the voltage sag is determined by distance from fault and supply voltage.
Table 1: Voltage distortion limits for harmonics

\begin{tabular}{lcc}
\hline Bus voltage & $\begin{array}{c}\text { Total harmonic } \\
\text { distortion for } \\
\text { individual voltage (\%) }\end{array}$ & $\begin{array}{c}\text { Total harmonic } \\
\text { distortion for } \\
\text { three-phase voltage (\%) }\end{array}$ \\
\hline $\mathrm{V}<69 \mathrm{kV}$ & 3.0 & 5.0 \\
$69 \mathrm{kV} \leq \mathrm{V}<61 \mathrm{kV}$ & 1.5 & 2.5 \\
$\mathrm{~V} \geq 161 \mathrm{kV}$ & 1.0 & 1.5 \\
\hline
\end{tabular}

Energizing the transformer: The medium voltage line contains many transformers. When operating this line these transformers energize at the same time which leads to a very high current flow. This causes the voltage drop for a short period. This drop is the voltage sag and its effect is obvious to consumers.

Starting of induction motor: Induction motors take a very high current in starting up about $>5-6$ times the nominal value taken by the motor. This high current reminds until the induction motor starts running with the nominal speed. The voltage drop depends on specifications of power system and the induction motor.

Voltage distortion limits IEEE: The voltage harmonic distortion limits apply to the quality of the power. For instance for systems of $<69 \mathrm{kV}$, IEEE 519 requires limits of $3 \%$ harmonic distortion for an individual frequency component and $5 \%$ for total harmonic distortion (Table 1 ).

Power quality improvement techniques and solutions: Power quality problems can be defined as the difference between the quality of power supplied and the quality of power required for reliable operation of the load equipment. Several types of power enhancement devices have been developed over the years to protect equipment from power disturbances. Some of the effective and economic measures can be identified as following. 
Power conditioning devices

Custom power devices

Power conditioning devices

Lightning and surge arrestors: Arrestors are using to protect the transformers from lightning and voltage surges but are certainly not sufficient for limiting voltage disturbances to protect sensitive electronic circuits from voltage surges.

Transient Voltage Surge Capacitors (TVSC): These units clamp spikes to a level that it is safe for the sensitive loads. Employing an entire facility protection strategy will safeguard the electrical system against most transients.

Filters: Provide protection against high frequency low voltage noises. Filters are designed to pass the fundamental frequency and reject the higher frequency noise such as Electromagnetic Interference (EMI) and Radio Frequency Interference (RFI). Harmonics filters prevent the harmonics content of non linear loads from back to the power source.

Isolation transformer: Provides a degree of filtering and isolation. Isolation transformers reduce electrical noise by separation of the primary and secondary through magnetic isolation. Isolation transformer reduce noises and harmonics but it does not compensate for power outages and voltage fluctuations.

Voltage regulators: Voltage regulators maintain output voltage at nominal voltage under severe input voltage variations. There are three basic types of regulators:

Tap changing transformer: Designed to adjust for varying voltages by automatically transferring taps on a power transformer. The main advantage of tap changers is high efficiency, wide input range, high over load current capability and good noise isolation compared to other voltage regulation technology. Disadvantages are noise created when changing taps and no waveform correction. The tap-changing transformer is: slow in response, exhibits contact erosion needs routine maintenance of its parts has an uneconomical size and requires frequent replacement of transformer oil (Woodley et al., 1999).

Buck boost: Utilizes similar technology to the changers except the transformer being not isolated. One of the advantages is that it can withstand high in-rush currents. Disadvantages are noise created when changing taps, poor noise isolation and no waveform correction.
Constant Voltage Transformer (CVT): It is also known as ferro resonant transformer. The CVT is a static regulator that maintains a nearly constant output voltage during large voltage variations in the input voltage. Advantages are superior noise isolation, very precise output voltage and current limiting for overload protection. The lack of moving parts means that the transformer requires little maintenance. Disadvantages are large size, audible noise and low efficiency.

Uninterruptible Power Supply (UPS): UPS systems provide protection in the case of a complete power interruption. There are three major UPS topologies each providing different levels of protection: off-line UPS, line interactive UPS and on-line UPS. Topology may be considered according to the load requirement based on efficiency, cost and transfer time. Moreover, UPS also requires a high level of maintenance because of leakage of batteries and also needs replacement for every 5 years.

Custom power devices: Customers are demanding electrical power with high quality from the electric utilities. Custom power devices are capable to solve power quality problems. The concept of custom power is based on the use of power electronic controllers in the distribution system for the purpose of providing reliable and high quality power that is needed by sensitive equipments to power quality variations.

Types of custom power devices: There are two major categories of custom power devices:

- Network reconfiguring type

- Compensating type

Network reconfiguring type (switchgear): It is used for power quality enhancement and these include: Static Current Breaker (SCB), Static Current Limiter (SCL) and Static Transfer Switch (STS).

Static Current Limiter (SCL): SCL limits a fault current by quickly inserting a series inductance in the fault path. It consists of a pair of anti-parallel gate turn off thyristors switch with snubbers ( $\mathrm{RC}$ circuit) and a current limiting inductor. The currents limiter is connected in series with a feeder such that it can restrict the current in the case of a fault downstream. In the healthy state, the opposite poled switch remains closed. These switches are opened 
Table 2: The types of network reconfiguring devices and their functions

\begin{tabular}{ll}
\hline Variables & Values \\
\hline Static Current Limiter (SCL) & Limits fault current \\
Static Circuit Breaker (SCB) & Disconnects the faulted circuit \\
Static Transfer Switch (STS) & $\begin{array}{l}\text { Avoid interruption, sag, swell by } \\
\text { connecting healthy feeder }\end{array}$ \\
\hline
\end{tabular}

when a fault is detected such that the fault current now flows through the current limiting inductor (Ghosh and Ledwich, 2002).

Static Circuit Breaker (SCB): SCB breaks a faulted circuit much faster than a mechanical circuit breaker. An SCB has almost the same topology as that of an SCL except that the limiting inductor is connected in series with an opposite poled thyristor pair. The Gate Turn Off thyristor (GTO) are the normal current carrying elements. The thyristor pair is switched on simultaneously as the bidirectional switch GTO is switched off once a fault is detected. This will force the fault current to flow through the limiting inductor. The thyristor pair is blocked after a few cycles if the fault still persists. The current through the thyristor pair will case to flow at the next available zero crossing of the current (Ghosh and Ledwich, 2002).

Solid-state switch based on the thyristor device (STS): The properties of a thyristor (ON-state and OFF-state) are used to perform an intelligent switch which can choose between two power sources and provide the best available power to the electrical load (Bhanoo, 1998). In most cases the STS is capable to limit the duration of voltage sags and interruptions to $<0.5$ cycle by transferring the loads from the affected feeder to a backup feeder. STS response is very high speed (Bongiorno et al., 2003) but when both the feeders are affected by voltage disturbances STS become not suitable. The reconfiguring types and its functions is shown in Table 2.

Compensating type: It is used for voltage regulation, power factor correction, load balancing and active filtering. Compensating type are include, Distributed Static Compensator (DSTATCOM), Dynamic Voltage Restorer (DVR) and Unified Power Quality Conditioner (UPQC).

Dynamic Voltage Restorer (DVR): DVR is a compensating custom power type device. Voltage Source Inverter (VSI) of DVR generates a compensating voltage which is then injected in the distribution system by means of series injection transformer. Passive filter connected
Table 3: The compensation type devices and their functions

\begin{tabular}{ll}
\hline Variables & Values \\
\hline Dynamic Voltage Restorer & Mitigate voltage sag, voltage \\
(DVR) & harmonics, voltage swell and flicker \\
Distributed Static Compensator & Bus voltage regulation, power factor \\
(DSTATCOM) & correction and harmonic filtering \\
Unified Power Quality Conditioner & Voltage regulation, reactive power \\
(UPQC) & compensation and harmonic filtering \\
\hline
\end{tabular}

between the VSI and the injection transformer, eliminates the higher order harmonic components from the inverter output voltage. Energy storage device connected to the VSI provides the necessary active power for the compensation (Bongiorno et al., 2003). DVR compensation ability depends on the range of sags and size of the energy storage.

DSTATCOM: Shunt devices are effective to compensate small voltage variation which can be controlled by reactive power injection. The ability to control the fundamental voltage at a certain point depends on the impedance to the supply and the power factor of the load. The compensation of a voltage dip by current injection is very difficult to achieve because the supply impedance is usually low and the injected current has to be very high to increase the load voltage.

Unified Power Quality Conditioner (UPQC): This system is a combination of a shunt controllers (DSTATCOM) and a series (DVR) these will be connected together by a common DC link capacitor, this combination facilitates the sharing of active power. It mitigates both load current and supply voltage problems simultaneously. Combining the series/shunt controllers increased the installed rating and improved the performance of the system but also costs will be higher. Table 3 shows the compensation types with its function.

\section{RESULTS AND DISCUSSION}

Without custom power devices: Figure 11 shows the Simulink Model of the test system in which the three parallel feeders are cleared. Fault are simulated for a periode of 1.5 from $0.8 \mathrm{sec}$ till $0.95 \mathrm{sec}$ at the first feeder.

Double line to ground fault: Double line to ground fault at the first feeder causes unsymmetrical sag at the adjacent feeder as shown in Fig. 12.

Three phase fault: Three phase fault at the first feeder causes a symmetrical sag at the neighboring feeder as depicated in Fig. 13. 


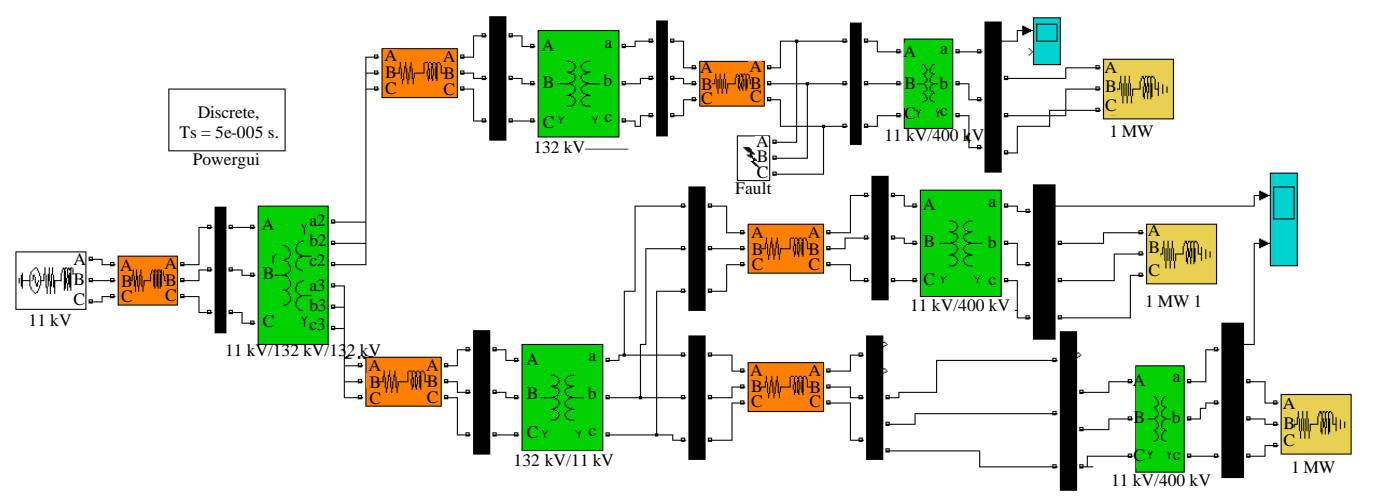

Fig. 11: Modeling and simulation the power system without custom power device
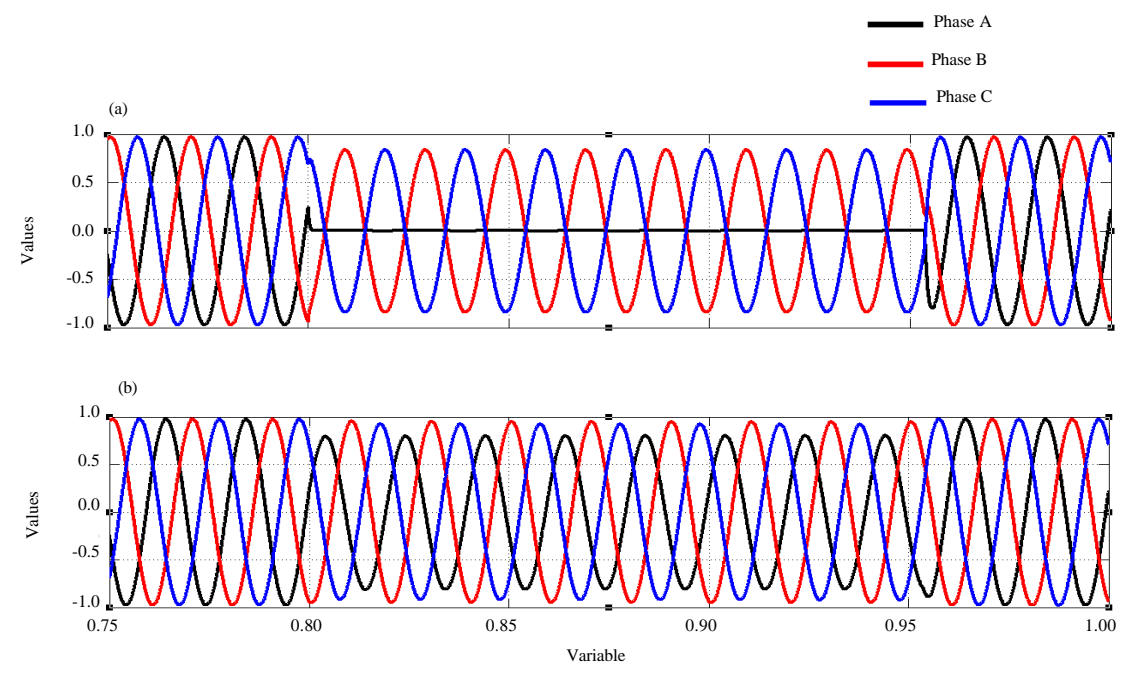

Fig. 12: a) Load voltage at thefirst feeder and b) Load voltage at the adjacent feeders
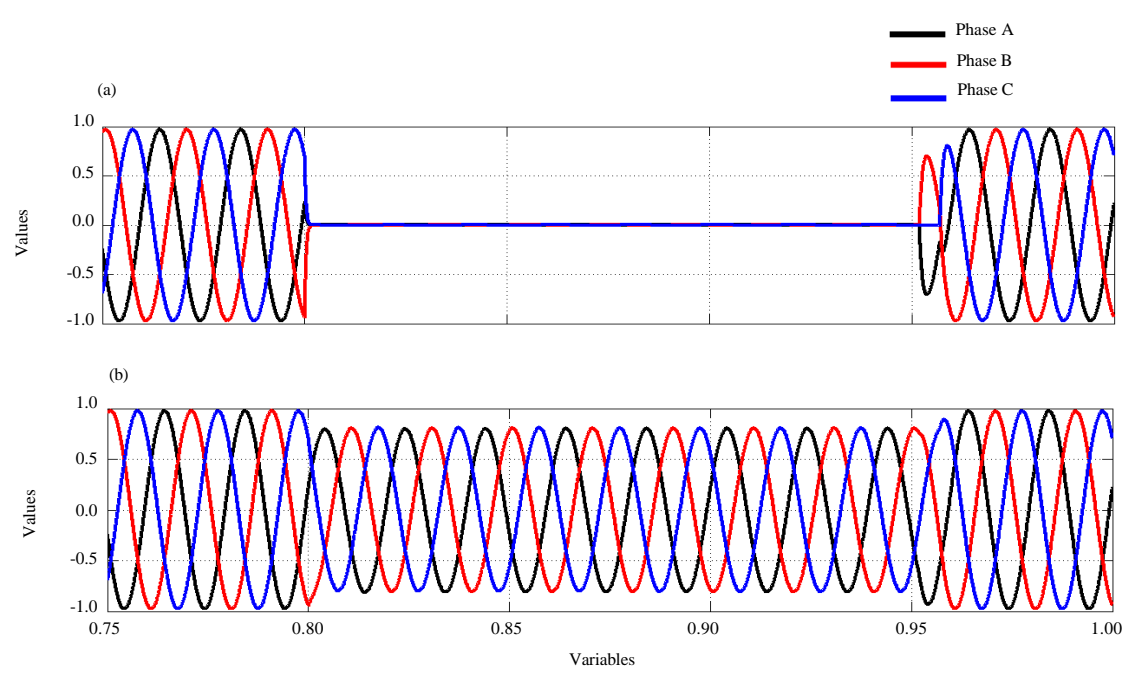

Fig. 13: a) Load voltage at the first feeder and b) Load voltage at the adjacent feeders 


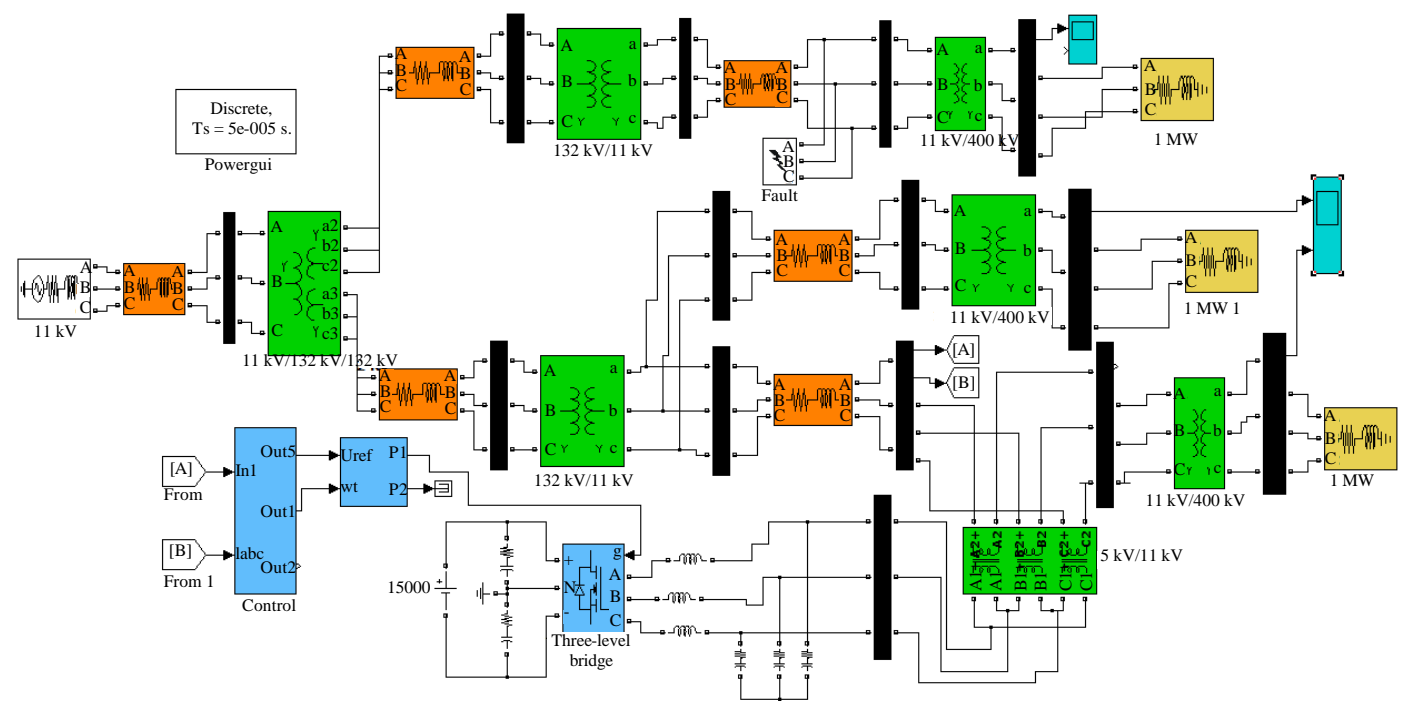

Fig. 14: Modeling and simulation the power system with a custom power device (DVR)

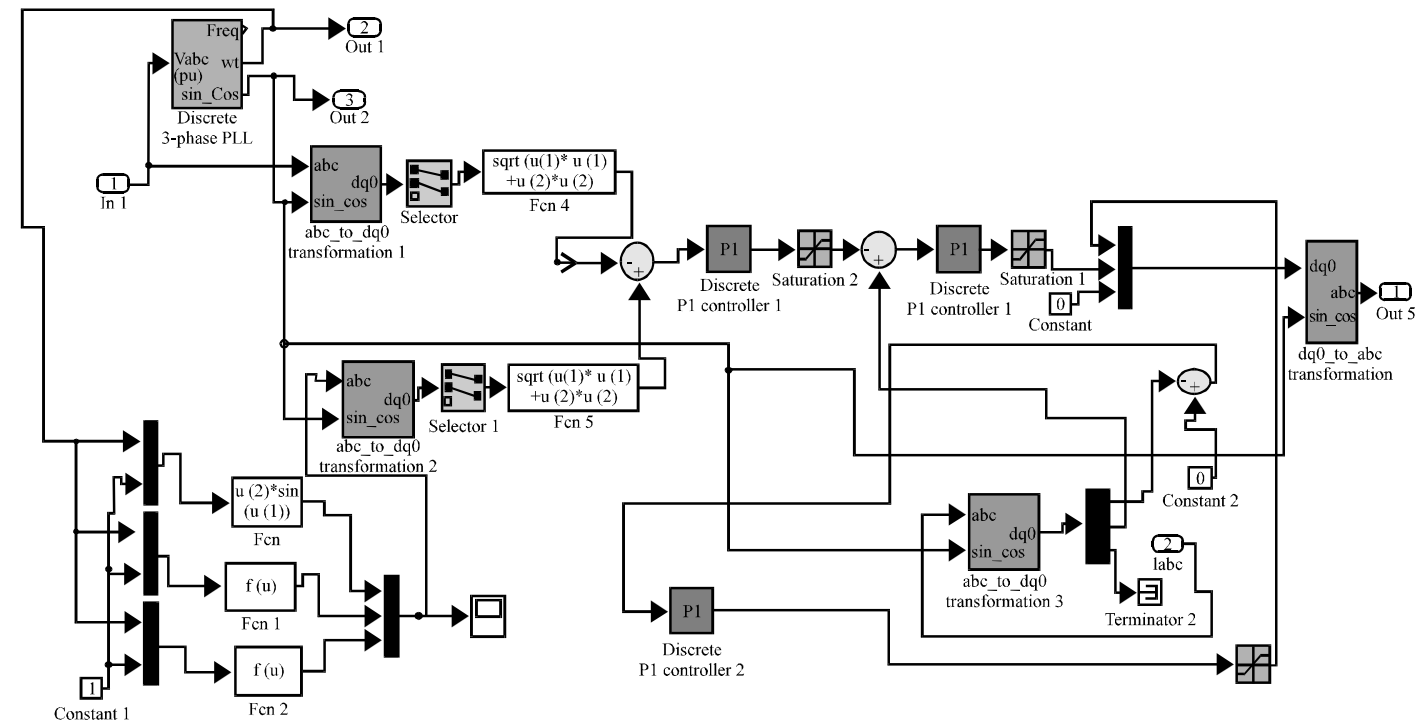

Fig. 15: Modeling and simulation the control system of the DVR based on PI controller

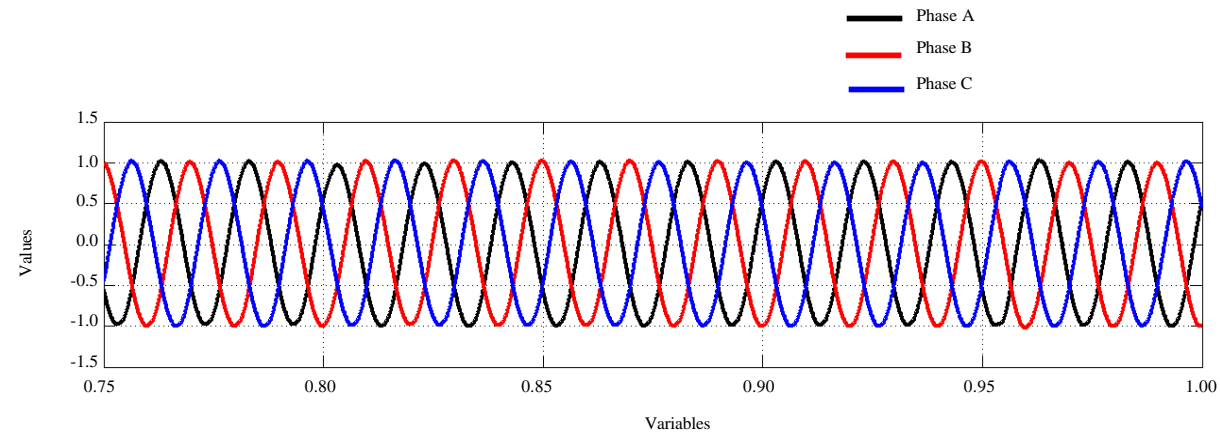

Fig. 16: Load voltage at the third feeder with custom power device (DVR) 


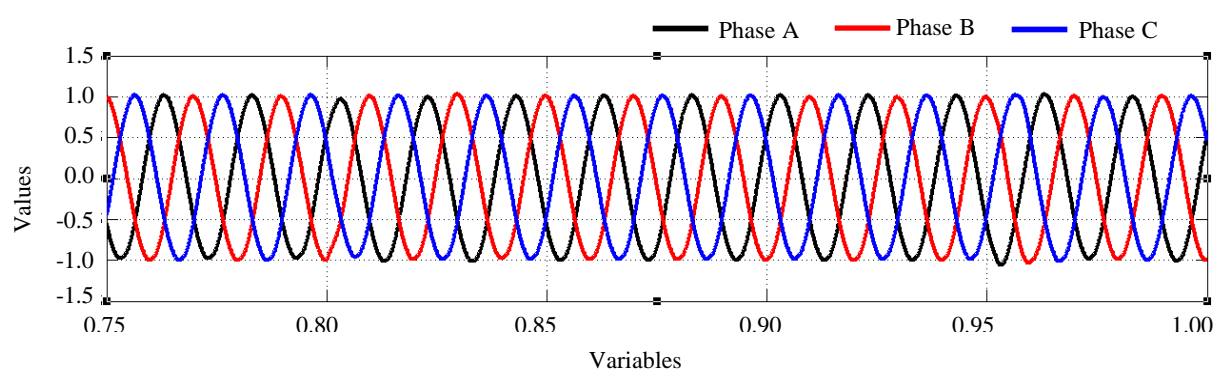

Fig. 17: Load voltage at the third feeder with custom power device (DVR)

With custom power device: Figure 13 shows the simulink model of the test system in which Dynamic Voltage Restorer (DVR) is connected at the third feeder. The control system of the DVR based on PI controller is demonstrated in Fig.14.

Double line to ground fault: Figure 15 shows that the custom power device (DVR) restores the load voltage to its pre-sag value.

Three phase fault: Figure 16 shows that the custom power device (DVR) restores the load voltage to its nominal value (Fig. 17).

\section{CONCLUSION}

With the increasing use of sensitive loads, power quality problems such as voltage sags, swells, flickers and harmonics are introduced into the distribution system. Voltage sag and harmonics are most common power problems in the industrial sectors. Loss of synchronization of processing equipment and lock ups of computer is a severe problem for continuous manufacturing industries. Circuit breakers tripping and data loss is the main problem caused by poor power quality in the service and transport sectors. The FACTS devices are used to improve the power transfer capabilities and stability margins of the transmission line. The custom power devices are effective to restore the sensitive load voltage to the pre-fault value and make it smooth under different cases of faults and nonlinear load condition. Some of these custom power devices include: DSTATCOM, UPQC and DVR, etc. Among these Dynamic Voltage Restorer (DVR) which is series connected power electronic based device is one of the most efficient and effective custom power devices to protect the sensitive equipments against voltage sags and harmonics due to its lower cost, smaller size and dynamic response. The simulation results showed that the load voltage was compensated fully within the permissible THD limit with the help of the dynamic voltage restorer which is one of the custom power devices types.

\section{ACKNOWLEDGEMENT}

This letter which Id write to show all my regards and respect to Asst. prof. D. wael H. Zayer i know him from his cleverness, ability to improve his scientific knowledge, intelligence and patience in all fields of his research. He was worked and pubrished many paper in many fierds as (Electrical power olmage processing, artificial intelligent and control).

\section{REFERENCES}

Babu, P.S. and N. Kamaraj, 2017. Power Quality Enhancement using Dynamic Voltage Restorer. Lap Co Inc., Ithaca, New York, USA., ISBN:109783330348622, Pages: 176.

Bhanoo, M.M., 1998. Static transfer switch: Advances in high speed solid-state transfer switches for critical power quality and reliability applications. Proceedings of the 1998 IEEE Annual International Conference on Textile, Fiber and Film Industry Technical, May 5-7, 1998, IEEE, Charlotte, North Carolina, USA., ISBN:0-7803-4962-8, pp: 1-8.

Bhattacharyya, S. and S. Cobben, 2011. Consequences of Poor Power Quality-An Overview. In: Power Quality, Eberhard, A. (Ed.)., InTech Open, London, England, UK., ISBN:978-953-307-180-0, pp: 1-25.

Bhattacharyya, S., J.M.A. Myrzik, J.F.G. Cobben, W.L. Kling and M. van Lumig et al., 2007. Need of voltage quality regulation in the future electricity infrastructure. Proceedings of the 9th International Conference on Electrical Power Quality and Utilisation (EPQU 2007), October 9-11, 2007, IEEE, Barcelona, Spain, ISBN:978-84-690-9441-9, pp: $1-6$. 
Bollen, M.H.J., 2001. Understanding Power Quality Problems: Voltage Sags and Interruptions. Vol. 445, IEEE Press, New York, USA., ISBN-13:9788186308844, Pages: 548.

Bongiorno, M., A. Sannino and L. Dusonchet, 2003. A cost-effective power quality improvement for industrial plants. Proceedings of IEEE International Conference on Power Technology Vol. 4, June 23-26, 2003, IEEE, Bologna, Italy, pp: 1-6.

Chan, K., A. Kara, P. Daehler, J. Guay and R. Tinggren, 1999. Innovative system solutions for power quality enhancement. Proceedings of the 15th International Conference on Electricity Distribution, June 1-4, 1999, AIM, Nice, France, pp: 2-25.

Ghosh, A. and G. Ledwich, 2002. Power Quality Enhancement Using Custom Power Devices. Kluwer Academic Publishers, London.
Manson, J. and R. Targosz, 2008. European power quality survey report. MSc Thesis, Leonardo Energy Initiative, USA.

Pohjanheimo, P., 2003. A probabilistic method for comprehensive voltage sag management in power distribution networks. Ph.D Thesis, Department Electrical and Communications Engineering, Helsinki University of Technology, Espoo, Finland.

Singh, B.N. and M. Simina, 2004. Intelligent solidstate voltage restorer for voltage swell/sag and harmonics. IEE. Proc. Electr. Power Appl., 151: 98106.

Woodley, N.H., L. Morgan and A. Sundaram, 1999. Experience with an inverter-based dynamic voltage restorer. IEEE Trans. Power Delivery, 14: 1181-1185. 